\title{
IDENTIFICACIÓN DE LOS PARÁMETROS DEL MODELO DEL NÚMERO DE CURVA Y SU INCERTIDUMBRE MENSUAL EN LA CUENCA ALTA DEL RÍO BOGOTÁ
}

\author{
IDENTIFYING PARAMETERS OF A CURVE MODEL NUMBER AND MONTHLY UNCERTAINTY AT \\ BOGOTA UPPER BASIN
}

Jorge Luis Corredor Rivera

Ing. Civil, Esp., Profesor Asistente, Facultad de Ingeniería

Universidad Militar Nueva Granada, Bogotá, Colombia

jorge.corredor@unimilitar.edu.co,vch@unimilitar.edu.co

Víctor Manuel Peñaranda Vélez

Ing. Civil, M.Sc., Profesor Asistente, Facultad de Ingeniería

Universidad Pontificia Bolivariana, Bucaramanga, Santander, Colombia

victor.penaranda@upb.edu.co

Fecha de recepción: 11 de noviembre de 2011

Fecha de aprobación: 03 de mayo de 2012

\section{RESUMEN}

Los estudios hidroclimatológicos implican manejar grandes volúmenes de información que pueden presentar interrupciones en la longitud de sus periodos de registro, datos anómalos, datos inconsistentes o errores en la transcripción de los datos en bases informáticas. Así mismo, la red hidroclimatológica no necesariamente presenta la mejor distribución en una región determinada. Además, existen zonas en donde no hay estaciones que permitan una interpretación local de la variación temporal y espacial de las variables climatológicas. Por lo anterior, es necesario acudir a técnicas de exploración de la información para encontrar las series que representen de la mejor manera, el comportamiento de una variable determinada para su aplicación en los procesos de modelación que sean necesarios. En este documento se presenta el alcance de una investigación en el campo de la modelación hidrológica, cuyo eje es la búsqueda para identificar los parámetros del modelo del número de curva y desarrollar un análisis de incertidumbre bajo el marco metodológico Generalized Likelihood Uncertainty Estimation (GLUE), con el propósito final de establecer los niveles de incertidumbre en los protocolos de modelación lluvia-escorrentía mensual sobre sistemas de cuenca con información escasa. Para desarrollar esta investigación, se seleccionó la cuenca alta del río Bogotá como sistema hidrológico de exploración, dado que cuenta con información hidrológica y climatológica suficiente y adecuada para implementar el modelo y el seguimiento de la metodología GLuE. Los resultados preliminares mostraron algunas dificultades del modelo para identificar algunos de sus parámetros. Sin embargo, la inclusión de un nuevo término en la base formal del modelo permitió visualizar la posibilidad de describir la 
dinámica de los sistemas de flujo de agua subterránea con la descripción del tipo, uso y cobertura del suelo con base en la selección rigurosa y adecuada del número de curva.

Palabras clave: GLUE, incertidumbre, número de curva.

\begin{abstract}
Hydro-meteorological studies involve large data volumes that may have disruptions related to length of their record periods, wrong data, inconsistent data, or data transcription errors to be included in databases. Likewise a hydro-meteorological network not necessarily offers the best arrangement in a given region. There are also areas where there is not any station to give a local interpretation about time and spatial variation of meteorological variables. Therefore, it is required to have information exploration techniques in order to find series representing the behavior of a particular variable for its application by modeling processes, if required. This article reports a research scope of hydrologic modeling focused upon an identification of parameters of a curve number model and a development of an uncertainty analysis following the methodological standard Generalized Likelihood Uncertainty Estimation GLUE) in order to establish the uncertainty levels of monthly rainfall-runoff modeling protocols upon hydrologic systems based on scarce information. To develop this research, Bogotá upper basin has been selected as the hydrologic system for exploration having enough and adjusted information to implement hydro-meteorological models and the GLUE. Preliminary results exhibit some issues to identify other relevant parameters; however, inclusion of a new variable in the standard model illustrates the possibility to explain the groundwater systems related to description of type, usage and land cover based on a strict and convenient curve number selection.
\end{abstract}

Keywords: GLUE, uncertainty, curve number.

\title{
INTRODUCCIÓN
}

Las complejas relaciones de los elementos que constituyen el entorno ambiental, en donde se enmarcan los recursos hídricos, hacen necesario establecer un protocolo de modelación exigente y detallado. No obstante, el empleo de los principios hidrológicos con respaldos más estrictos, usando modelos más robustos que hay en la literatura científica, acordes con el desarrollo de la tecnología y el avance del conocimiento en algunas de las firmas consultoras de Colombia, es relativamente reciente e incipiente, función que en cierto modo, se delega a la universidad [1]. Por otra parte, la práctica de la ingeniería se ha venido ajustando a un conjunto de paradigmas contemporáneos que simplifican en forma considerable, los esfuerzos por proveer una razón holística que explique los procesos complejos en hidrología. 
Una realidad nacional es la ausencia de información suficiente en diferentes tipos de análisis hidroclimatológicos, aunque buena parte de esta información existe desde 1970, y aunque también hay que reconocer la discontinuidad de los periodos de registro que pueden darse en algunas estaciones de red hidroclimatológica disponible. La ausencia de información sobre mediciones de corta duración para las variables hidroclimatológicas es mucho mayor.

Por lo anterior, es necesario emplear metodologías simplificadas en la solución de proyectos relacionados con la planeación, la gestión y el manejo de los recursos hídricos, considerando las limitantes de sus implementaciones para obtener resultados tangibles sobre los niveles de incertidumbre a los cuales pueden estar expuestos esta clase de proyectos.

En este trabajo, se plantea una reflexión al respecto y se seleccionó una de las metodologías más aceptadas en el marco de la modelación hidrológica que se conoce como Teoría del Número de Curva (CN, sigla en inglés de Curve Number). Esta metodología parte de una base conceptual empírica que se ha venido fortaleciendo en los últimos años con la adecuación de la misma desde un marco agregado (por ejemplo: se analiza el sistema bajo una única unidad de respuesta), hasta constituirse en una herramienta para la modelación distribuida (por ejemplo, el proceso hidrológico es analizado en diferentes sitios dentro de la escala espacial), tal como son los modelos: HEC-HMS, EPA SWMM, SWAT, SPUR-91, CREAMS, entre otros Shing [25].

La Teoría del Número de Curva se aplica en la evaluación de crecientes, considerando tres variables relevantes: (i) la precipitación que corresponde a valor de la cantidad de lluvia estimada en una cuenca de interés, (ii) la humedad anterior, concepto que se identifica con la cantidad de Iluvia acumulada antes de ocurrir la lluvia considerada para la cual se establece un período que puede oscilar entre 5 a 30 días, y (iii) el complejo de suelo vegetación que asocia a la evaluación de la escorrentía directa, el uso del suelo y la cobertura vegetal [2]. Si se tiene en cuenta otras metodologías para evaluar el balance hídrico con fines de riego, la escala de tiempo puede ser semanal, decadal o mensual; en consecuencia, cabe la posibilidad de examinar el comportamiento de una determinada metodología en diferentes escalas de tiempo, motivo por el cual para este trabajo, se eligió la escala de tiempo mensual.

A partir del modelo del Número de Curva, se presenta una breve discusión sobre la complejidad del procedimiento de calibración y más aún, sobre la incertidumbre en la estimación de sus parámetros que conforman tal modelo, para lo cual fue necesario hacer algunas modificaciones en la base formal del modelo, introduciendo un nuevo término en la ecuación funcional del mismo y verificando su identificabilidad por medio del marco metodológico Generalized Likelihood Uncertainty Estimation (GLUE). Algunos investigadores han empleado la metodología GLUE para dar soporte y confianza en las predicciones de la modelación hidrológica [3, 4], en este caso, se utilizó un procedimiento similar para analizar los resultados en la evaluación de la base conceptual propuesta y así, esclarecer las bondades del modelo planteado y los niveles de incertidumbre a los cuales se somete quien lo implemente. 


\section{1. ÁREA DE INFLUENCIA DEL PROYECTO}

La región seleccionada corresponde a la cuenca alta del río Bogotá hasta la estación hidrométrica Saucio, cuya cuenca constituye el área de influencia para este proyecto que comprende un área de $272.3 \mathrm{~km}^{2}$, se localiza sobre el altiplano cundiboyacence en el espacio geográfico definido principalmente por los municipios de Turmequé (Boyacá), Villapinzón y Chocontá (Cundinamarca), delimitado al norte por el municipio de Turmequé, al sur por el embalse del Sisga, al oriente por el municipio de La Capilla y al occidente por la laguna de Suesca.
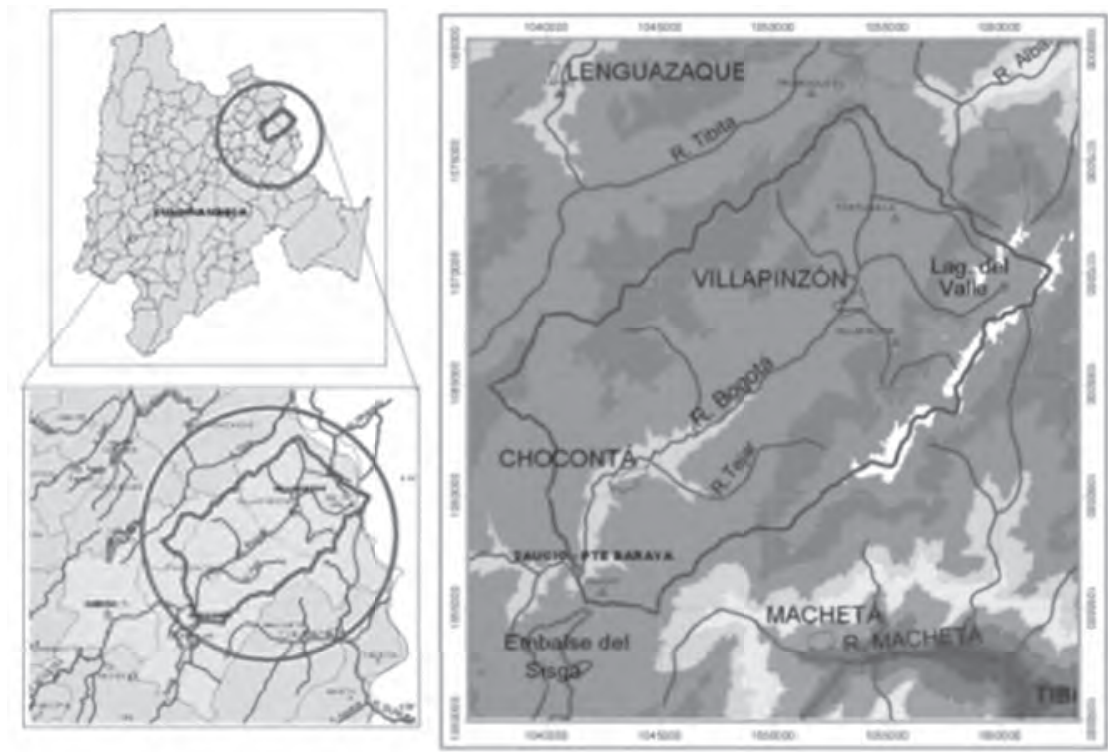

Figura 1. Cuenca del río Bogotá hasta Saucio

Fuente: Corporación Autónoma Regional de Cundinamarca, 2010

En la Figura 1, se muestra el área de influencia para la estación Saucio, que se seleccionó por su importancia en los contextos social y de impacto ambiental [5], y teniendo en cuenta que la influencia antrópica es relativamente baja. Por lo tanto, la demanda de agua sobre la cuenca todavía no ha producido alteraciones significativas sobre la disponibilidad hídrica en el rio. Desafortunadamente, el drenaje de los sistemas de riego y los vertimientos incontrolados y sin tratamiento previo de las aguas residuales de algunas curtiembres y otros tipos de vertimientos, han hecho que la calidad del agua del río presente niveles muy bajos para el sistema ecológico y el entorno ambiental local.

Desde luego, la prioridad del empleo del modelo del CN se estableció en los planes de ordenamiento de cuencas y reglamentación de corrientes que tienen especial interés en las Corporaciones Autónomas Regionales (CAR). Por lo anterior, es necesario caracterizar los patrones hidrológicos sobre esta región geográfica, para poder ofrecer una propuesta tendiente a mejorar las prácticas de planeamiento, gestión y manejo del recurso hídrico. 


\section{INFORMACIÓN DISPONIBLE}

En la Tabla 1, se relacionan las estaciones utilizadas en este proyecto, cuya selección obedeció entre otros, a los siguientes factores: (i) vecindad con el área de influencia del proyecto, (ii) disponibilidad de información, con el fin de caracterizar el régimen pluvial local, (iii) facilidad para establecer los parámetros que exigen las metodologías de cálculo consideradas con un rango de aceptabilidad razonable, teniendo en cuenta que la aplicación de tales metodologías se restringe a un estado estacionario. La información disponible presenta un periodo de operación de unos 61 años, suficiente para comprender la dinámica temporal de las variables hidroclimatológicas necesarias. Los registros de precipitación cumplen con la condición de continuidad, es decir, no hay interrupciones en las observaciones.

Tabla 1. Red hidrometeorológica

\begin{tabular}{|c|c|c|c|c|c|c|}
\hline NOMBRE & TIPO & CÓDIGO & MUNICIPIO & ELEVACIÓN & ENTIDAD & $\begin{array}{c}\text { FECHA } \\
\text { INSTALACIÓN }\end{array}$ \\
\hline Saucio & LG & 2120719 & Chocontá & 2618 & CAR & $11 / 1940$ \\
\hline Saucio & PG & 2120027 & Chocontá & 2670 & CAR & $06 / 1947$ \\
\hline
\end{tabular}

Localmente y con base en la información de 15 estaciones con registros pluviales suministrados por el Instituto de Hidrología, Meteorología y Estudios Ambientales (IDEAM) y la CAR, se utilizó la metodología Inverse Distance Weight (IDW), implícita en ArcGis Versión 10, para analizar la distribución espacial de la precipitación media total mensual y anual, con el fin de conocer su comportamiento.

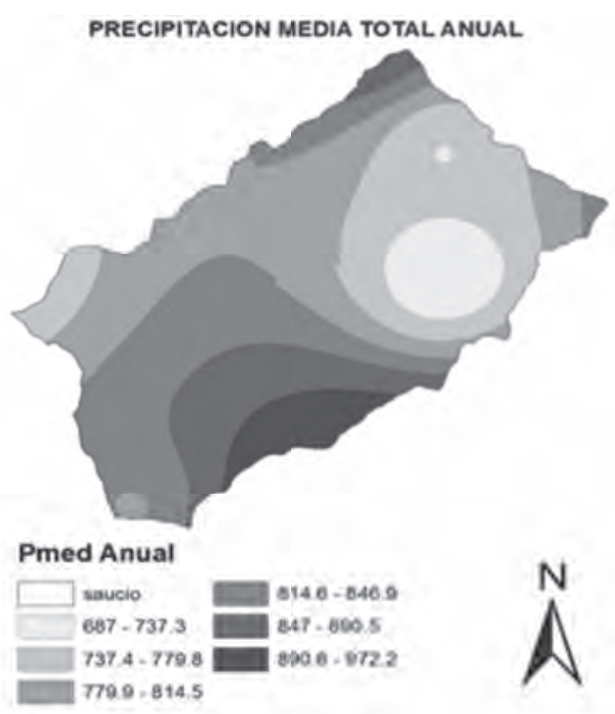

Figura 2. Distribución espacial del la precipitación media total anual multianual

Fuente: Los autores 
En la Figura 2, se muestra la distribución espacial de la precipitación media total anual. En general, se observa un sector al suroccidente que presenta una zona de mayor precipitación que desciende hacia el noroccidente con un sector de baja precipitación en la zona nororiental.

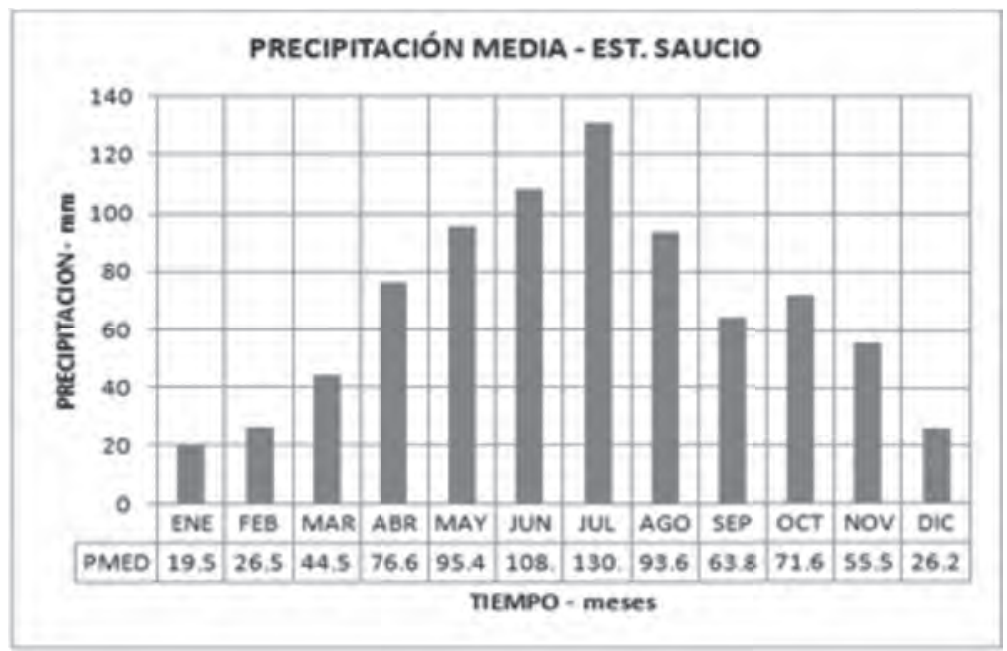

Figura 3. Distribución temporal de la precipitación media total mensual - Est. Saucio.

Fuente: Los autores

Para los propósitos de este proyecto, se seleccionó la estación pluviográfica Saucio con datos disponibles para el periodo de registro comprendido entre 1948 y 2003, cuyo valor de precipitación media total anual es de 812.6 mm/año, con una oscilación mensual entre 19.5 mm/mes y 130.8 $\mathrm{mm} / \mathrm{mes}$, con un valor máximo en 24 horas de $85 \mathrm{~mm}$, con la distribución temporal que se señala en la Figura 3. El número de días con precipitación al año es de 164, con una oscilación para los valores medio entre 6 y 20 días al mes.

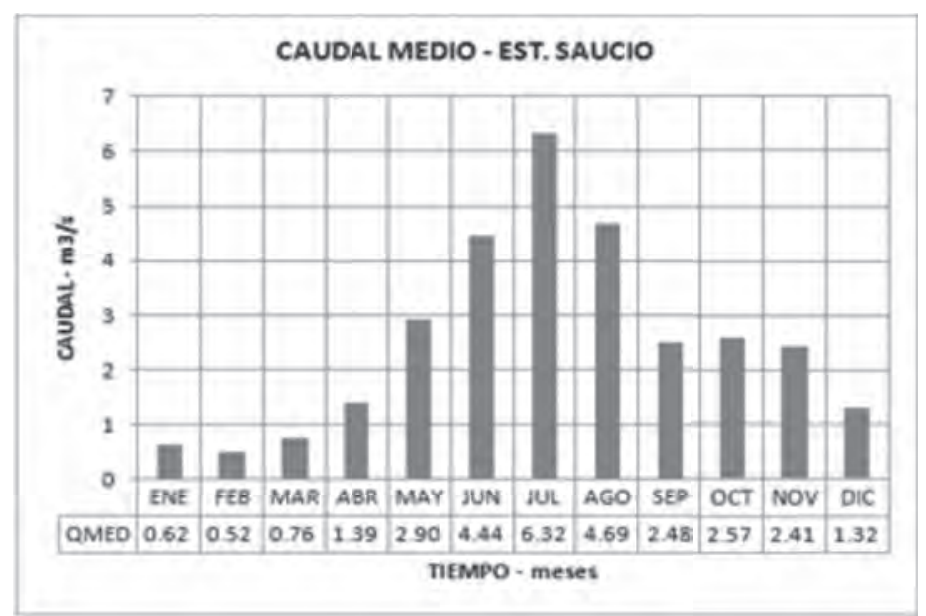

Figura 4. Distribución temporal del caudal medio mensual mutianual - Est. Saucio

Fuente: Los autores 
Como se mencionó, la estación liminigráfica seleccionada corresponde a la estación Saucio con un periodo de registro disponible comprendido entre 1943 y 2003, en el cual se registró un caudal medio mensual multianual de $2.54 \mathrm{~m}^{3} / \mathrm{s}$, un caudal mínimo mensual de $0.04 \mathrm{~m}^{3} / \mathrm{s}$ y un valor máximo mensual de $64.2 \mathrm{~m}^{3} / \mathrm{s}$ del río Bogotá. En la Figura 4, se muestra la variación temporal de los valores medios mensuales multianuales de los caudales cuya distribución responde con el comportamiento de la precipitación en períodos similares.

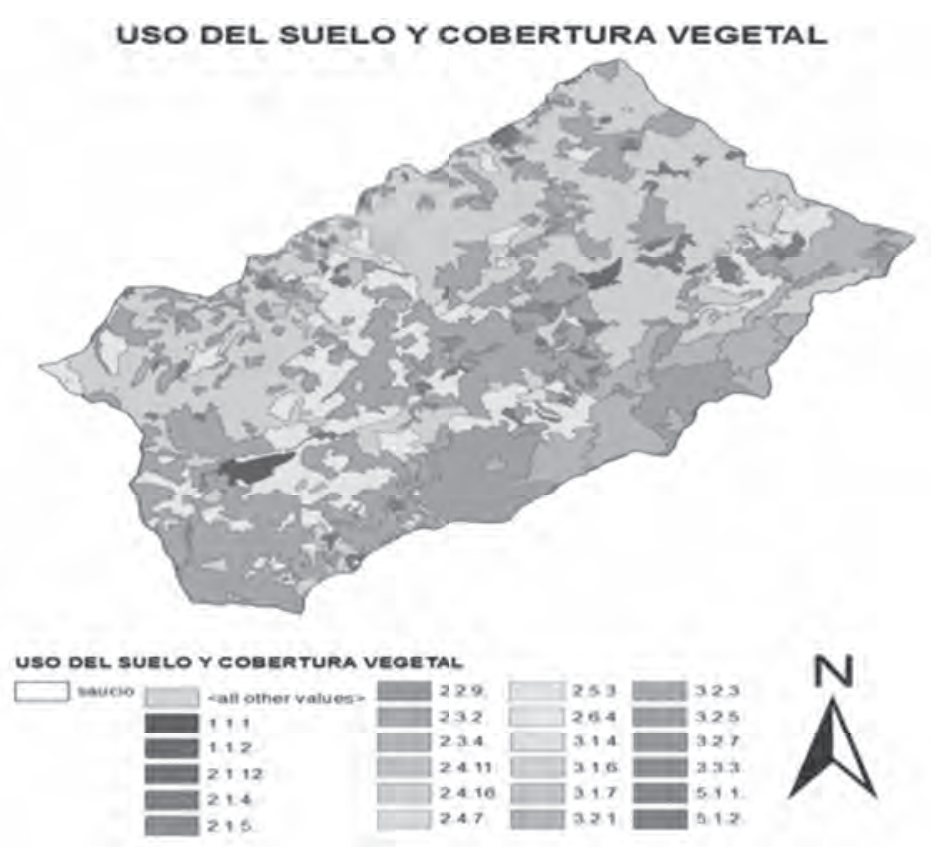

Figura 5. Uso del suelo y cobertura vegetal

Fuente: CAR

En la Figura 5, se muestran los usos del suelo y las coberturas vegetales identificadas en el área de influencia del proyecto [6]. Estas coberturas fueron clasificadas con la metodología Corine Land Cover, según la codificación que se indica en la misma Figura. En el uso del suelo y cobertura vegetal se encuentra tejido urbano, cultivos de papa, maíz, guanábana, cebada, cultivos en viveros, pastos, bosques y mosaicos de bosques, vegetación de páramo y subpáramo, tierras eriales y cuerpos de agua.

El tipo de suelo que se identificó [6] para el área de interés en este proyecto, es el que se muestra en la Figura 6. El relieve se presenta con pendientes entre el 5\% y el 75\% en un paisaje de montaña; en estos suelos, dominan las texturas finas a moderadamente finas, normalmente bien drenados y con fertilidad moderada a baja. 


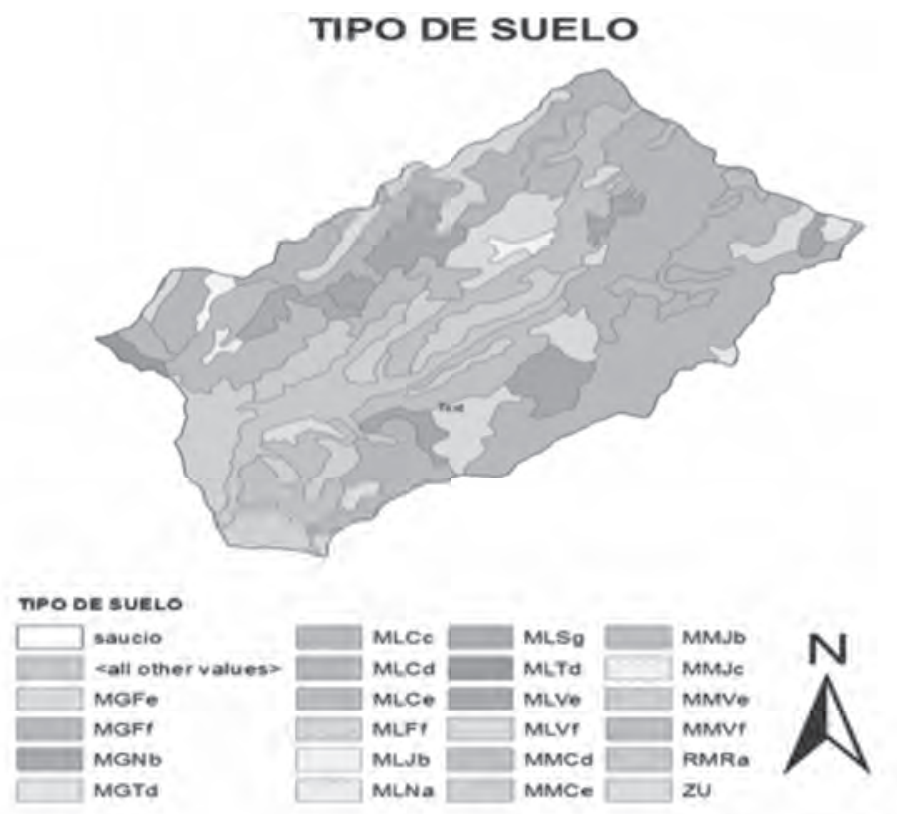

Figura 6. Tipo de suelo

Fuente: CAR

\section{MODELO DEL NÚMERO DE CURVA}

De las variables climatológicas, la precipitación es la de mayor interés debido a su influencia en la evaluación de caudales para el diseño de obras hidráulicas y oferta hídrica de una cuenca. De la precipitación total que ocurre sobre una cuenca, sólo una parte de ella llega al cauce de los ríos o a sistemas lénticos, lo cual se conoce como escorrentía directa o precipitación efectiva.

Hacia mediados de la década de los 50, el Servicio de Conservación de Suelos del Departamento de Agricultura de los Estados Unidos [7] desarrolló un procedimiento conocido como la Teoría de Número de Curva (CN), que considera, como ya se mencionó, las variables: (i) la precipitación, representada en este caso por la cantidad de lluvia que ocurre en un período previamente seleccionado; (ii) el complejo de suelo - hidrológico que considera la interrelación suelo - cobertura vegetal, y (iii) la condición de humedad antecedente; de acuerdo con estas variables, se fija un número de curva $(\mathrm{CN})$ que representa tal interrelación. Esta metodología fue originalmente propuesta en Chow [10] para evaluar la precipitación neta que podría generar una tormenta, considerando el volumen de precipitación antecedente en un período de 5 a 30 días [2], con el fin de establecer el escurrimiento directo que puede esperarse como respuesta a una precipitación especifica. Según este procedimiento, la escorrentía directa (Q) o precipitación efectiva, se expresa mediante la ecuación (1): 


$$
Q=\frac{\left(P-I_{a}\right)^{2}}{P-I_{a}+S}
$$

Que también se puede escribir como:

$$
Q=\frac{(P-0.2 S)^{2}}{(P+0.85)}
$$

Donde, las variables expresadas en unidades de altura de precipitación, se identifican como:

Q: Es la escorrentía directa o precipitación efectiva, $\mathrm{mm}$

P: Es la precipitación considerada, $\mathrm{mm}$

S: Es la diferencia potencial máxima entre P y $\mathrm{O}$ a la hora cuando se inicia la tormenta, y representa proporcionalmente la pérdida de escorrentía $I_{a}$ por infiltración, intercepción y almacenamiento superficial, $\mathrm{mm}$.

El valor de $I_{a}$ representa la intercepción, infiltración y almacenamiento superficial [2], el cual, mediante un arreglo matemático, se explica en la ecuación (2) con los valores 0.2 y 0.8 obtenidos de datos de cuencas grandes y pequeñas para Estados Unidos, motivo por el cual sus autores recomiendan que no se modifiquen [2]. En la ecuación anterior, el valor de S se determina a partir de la ecuación (3).

$$
S=\frac{25400}{C N}-254
$$

Donde: $\mathrm{CN}$ corresponde al valor del Número de Curva que se obtiene como una función del tipo de cobertura vegetal, uso del suelo y pendiente del terreno.

Dada la complejidad de las variables que implica esta metodología, la calibración de los resultados obtenidos y su aplicación, puede ser dispendiosa y para algunos autores, da lugar a dudas sobre su plena aceptabilidad [8], si se tiene en cuenta que las relaciones matemáticas que se hacen para obtener las ecuaciones se puede optimizar.

Colombia, por encontrarse en la zona tórrida, es una región tropical, por lo cual al aplicar esta metodología, se plantean algunas inquietudes, de carácter general y otras de tipo específico.

Precipitación. Una primera inquietud se plantea al considerar que esta metodología fue establecida para utilizar información diaria con fines de evaluación de crecientes, es decir, para escalas de tiempo de corta duración. Su posible aplicación a una escala de tiempo mensual no parece atractiva, pero la consideración de su aplicación a esta escala de tiempo puede ser útil cuando se trata de hacer modelaciones hidrológicas en zonas en donde no se dispone de información puede ser obtenida a partir de la espacialización de los datos puntuales, con la utilización de medios informáticos como el Arc-GIS. 
Otros autores han tratado el manejo de la precipitación total mensual para establecer una relación entre este valor y su desagregación diaria, como la propuesta de Dal-Ré y otros [9], que utilizan el concepto de precipitación virtual, según el cual la precipitación diaria se puede expresar en términos de la precipitación total mensual, la lluvia máxima diaria ocurrida en ese mes y el número de días con registros de lluvia, utilizando diferentes tipologías en la interacción de las variables mencionadas. Aunque en este trabajo no se hace alguna desagregación, es interesante mencionar que hay otros trabajos en los cuales se utilizan valores medios (virtuales), de precipitación total mensual para encontrar caudales medios mensuales y anuales.

Uso del suelo y cobertura vegetal. Una segunda inquietud planteada, es la validez del porcentaje de $I_{a}$ para zonas tropicales. La propuesta original considera que $S=0.2 I_{a^{\prime}}$ el valor de 0.2 es el sugerido por los autores de la ecuación [7] y representa el porcentaje de $S$ que ellos encontraron en términos de las regiones que estudiaron para establecer tal ecuación, y al mismo tiempo, recomiendan que no se modifique.

Para estudiar otros valores del porcentaje mencionado, se sustituyó el valor de 0.2 por $k$, denominado para los fines de este trabajo como factor de abstracción inicial, lo cual hizo que la ecuación también se escriba como:

$$
Q=\frac{(P-k S)^{2}}{(P+S-k S)}
$$

Condición de humedad antecedente. Para la condición de humedad antecedente, se estableció el rango de variación que se indica en la Tabla 2, de acuerdo con la cantidad de lluvia acumulada en los cinco días anteriores a la ocurrencia de la lluvia máxima en 24 horas, pero también se acepta que este período se extienda hasta 30 días $[2,10]$.

Tabla 2. Rango de variación para la condición de humedad antecedente -SCS

\begin{tabular}{|c|c|c|}
\hline \multirow{2}{*}{ CONDICIÓN ANTECEDENTE } & \multicolumn{2}{|c|}{ LLUVIA ANTECEDENTE (mm) } \\
\cline { 2 - 3 } & Invierno & Verano \\
\hline I & menor 35.6 & menor 12.7 \\
\hline II & $35.6-55.3$ & $12.7-27.9$ \\
\hline III & $55.3-$ & $27.9-$ \\
\hline Fuente: Chow Ven Te, Maidment D., Mays L. Applied Hidrology, 1988, $153 \mathrm{p}$.
\end{tabular}

La condición antecedente I es aquella en la cual los suelos están secos debido a la condición de verano o época de estiaje en una región determinada, sin llegar al punto de marchitez en las plantas; la condición II, para un estado climatológico promedio, y la condición III para períodos de mayor pluviosidad.

En Colombia, la mayor proporción de la información pluviométrica disponible más accesible que se utiliza, corresponde a los valores totales mensuales de precipitación. Por tal motivo, se podría 
establecer una modificación al modelo conceptual general, ajustando los términos del modelo a la escala mensual.

En este proyecto se sugiere entonces, describir los procesos lluvia - escorrentía a partir de la información pluviométrica (valores a la escala mensual de observación), e hidrométrica (valores medios mensuales observados), disponible. Para ello, se realizó un ajuste simple a la ecuación (4), considerando el aporte del flujo base $\left(Q_{b}\right)$, expresado en $\mathrm{mm} / \mathrm{mes}$, en los términos de la ecuación general, de modo que se obtuvo una expresión de la siguiente forma:

$$
Q=\frac{(P-k S)^{2}}{(P+S-k S)}+Q_{b}
$$

Donde, $Q_{b}$ es el caudal que se encuentra en forma permanente en el cauce del río en cualquier época del año, cuyo origen puede ser el aporte que genera el perfil hidrogeológico de la cuenca y el abastecimiento que suministran las zonas de páramo en los sectores más altos de la cuenca, cuando hay lugar para ello. Este aporte se agrega al flujo superficial directo que procede de la cuenca hidrográfica.

Dado que se desconoce la interrelación entre el parámetro $Q_{b}$ y los demás términos conjugados en la metodología del número de curva $Q_{b^{\prime}}$ se identifica aquí como una variable independiente que requiere para su estimación, considerarse como un parámetro fijo en la ecuación (5), sujeto a un procedimiento de calibración automática para su determinación.

\section{PROCEDIMIENTO DE CALIBRACIÓN OBJETIVA}

La calibración objetiva está basada en el criterio de minimización de algunos parámetros relacionados con los resultados que se esperan al aplicar el modelo del Número de Curva, y son: el factor de abstracción inicial $k$, el valor medio ponderado del Número de Curva (CN), para la condición promedio de humedad antecedente, y el valor medio de aporte del flujo base $\left(Q_{b}\right)$.

Para ejecutar el procedimiento de calibración objetiva, se empleó el algoritmo Shuffle Complex Evolution (SCE) [11], como herramienta para calibrar los parámetros del modelo CN a los valores observados en la estación hidrométrica Saucio.

Tabla 3. Parámetros de entrada del modelo CN muestreados por el SCE

\begin{tabular}{|c|c|c|}
\hline PARÁMETRO & DEFINICIÓN & RANGO DE BÚSOUEDA \\
\hline $\mathrm{k}$ & factor de abstracción inicial & $0.0-0.5$ \\
\hline $\mathrm{CN}$ & valor medio ponderado del CN & $0-100$ \\
\hline $\mathrm{Ob}$ & valor medio de aporte del flujo base & $-200-200 \mathrm{~mm} / \mathrm{mes}$ \\
\hline
\end{tabular}


Los rangos de búsqueda de los valores de los parámetros fueron seleccionados de acuerdo con los espacios posibles para la misma, los cuales fueron establecidos por investigaciones previas [12]. El conjunto de valores aleatorios de los parámetros se compone de 72.591 muestreos en los intervalos de los rangos indicados en la Tabla 3.

El análisis de identificación e incertidumbre de los parámetros del modelo del CN, se desarrolló desde el marco metodológico GLUE [13-15]. El índice de bondad de ajuste $\left(R^{2}\right)$ [16] empleado como función objetivo para evaluar el rendimiento y la medida de verosimilitud para el análisis GLUE, se expresa mediante la ecuación (6).

$$
L(S \mid \psi) \propto R^{2}=\frac{\sum_{i=1}^{N}\left(S_{i}-O_{i}\right)^{2}}{\sum_{i=1}^{N}\left(\left|S_{i}-\bar{S}\right|^{\prime}+\mid O_{i}-\overline{0}\right)^{2}}
$$

Donde $S$ es la variable simulada, $\psi$ representa al conjunto de parámetros para calibración, y $O$ es la variable observada. En la ecuación (6) los valores promedios fueron señalados con una barra sobre el nombre de la variable.

\section{RESULTADOS Y ANÁLISIS}

A continuación, se presentan los resultados más relevantes de la aplicación del modelo modificado.

\subsection{COMPLEJIDAD DEL ESPACIO DE BÚSOUEDA}

Con el propósito de establecer los niveles de complejidad del procedimiento de calibración automática, fue necesario analizar el comportamiento de los espacios de búsqueda tal y como sugiere Beven [1 7], para la modelación de sistemas ambientales. Dado que el modelo del Número de Curva se compone de tres (3) parámetros de entrada, se dificulta la revisión directa de la interrelación entre los parámetros del modelo y la evaluación de rendimiento del mismo. Teniendo en cuenta lo indicado anteriormente, se procedió a la construcción gráfica bidimensional de los espacios del modelo, ajustando con un valor fijo, alguno de los tres parámetros que conforman el modelo del número de curva.

En la Figura 7, se identifica dos espacios posibles del modelo, relacionando dos parámetros del modelo y el estimador de rendimiento RMSE (del Inglés, Root Mean Square Error). Tal como se puede apreciar, el espacio del modelo se puede representar por una superficie suavizada que muestra una clara inflexión y que permite en principio, dar a conocer un sistema sencillo del espacio de búsqueda de los parámetros del modelo. No obstante, la respuesta gráfica del modelo presenta una dificultad notable que se relaciona con la caída asintótica de la superficie de inflexión. En vista de tal situación, es factible establecer a priori, que los parámetros del modelo del número de curva no son identificables. 


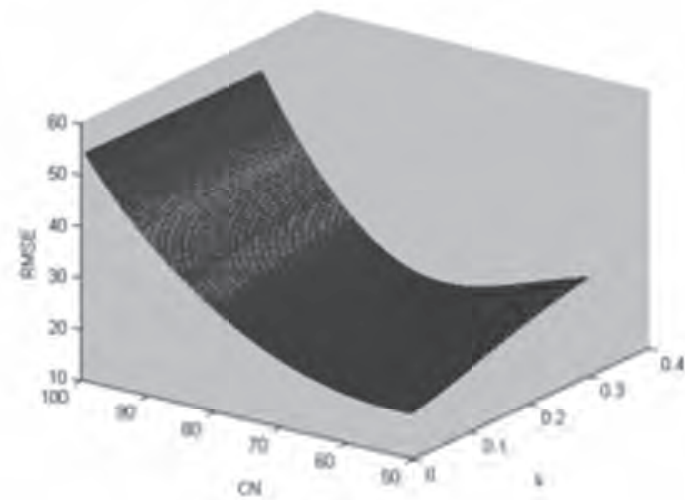

a)

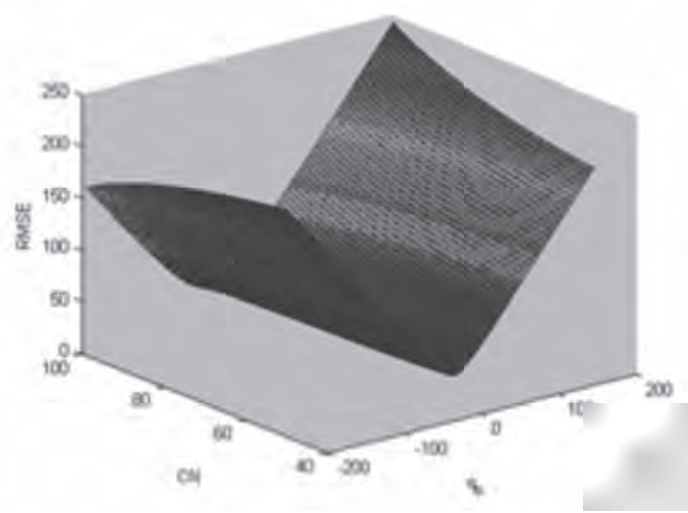

b)

Figura 7. Identificación de los espacios del modelo. a) Relación entre los parámetros CN y k. b) Relación entre los parámetros $\mathrm{CN} \mathrm{y} \mathrm{O}_{\mathrm{b}}$.

Fuente: Los autores

\subsection{RENDIMIENTO DEL MODELO DEL NÚMERO DE CURVA}

El rendimiento del modelo se presenta en la Tabla 4, donde 27.362 de los 72.591 conjuntos de parámetros presentan un rendimiento del $R^{2}$ de 0.68 . El mejor ajuste del modelo presenta un rendimiento del $R^{2}$ de 0.80 que a su vez, también se representa por el RMSE más bajo por valor de $17.65 \mathrm{~mm}$. Como se aprecia en los resultados, el rendimiento general del modelo es regular, pero los grupos de parámetros que presentan el mejor rendimiento, alcanzan un valor aceptable del $R^{2}$ en comparación con la eficiencia de otros modelos presentados en la literatura $[18,19]$.

Tabla 4. Resultados de rendimiento del modelo del CN seleccionado para el conjunto de datos observados

\begin{tabular}{|c|c|c|c|c|c|}
\hline DATOS & $\mathbf{k}$ & $\mathbf{Q}_{\mathbf{b}}$ & $\mathbf{C N}$ & $\mathbf{R}^{\mathbf{2}}$ & RMSE \\
& $\mathbf{I}-\mathbf{I}$ & $\mathbf{( m m )}$ & $\mathbf{I}-\mathbf{I}$ & $\mathbf{I}-\mathbf{I}$ & $\mathbf{( m m )}$ \\
\hline Mejor Ajuste & 0.07 & 8.48 & 57.06 & 0.80 & 17.65 \\
\hline Comportamiento promedio & 0.13 & -2.31 & 73.68 & 0.68 & 26.80 \\
\hline
\end{tabular}

\subsection{COMPORTAMIENTO DE LOS PARÁMETROS Y EOUIFINALIDAD}

En la Figura 8, se aprecian los diagramas de dispersión de los tres parámetros del modelo del número de curva, los cuales ilustran a $\mathrm{O}_{b}$ y CN como parámetros identificables y a k como un parámetro no identificable. Este último parámetro no identificable se distribuye en un amplio rango del espacio 
de muestreo con un comportamiento que dificulta su estimación y que refuerza el concepto de equifinalidad (parcial), de los parámetros del modelo. Siguiendo el concepto indicado por Beven [13] sobre la tesis de equifinalidad, el modelo presenta varias de las realizaciones aceptables para simular la respuesta del mismo, y establece un notable grado de incertidumbre en su aplicación.
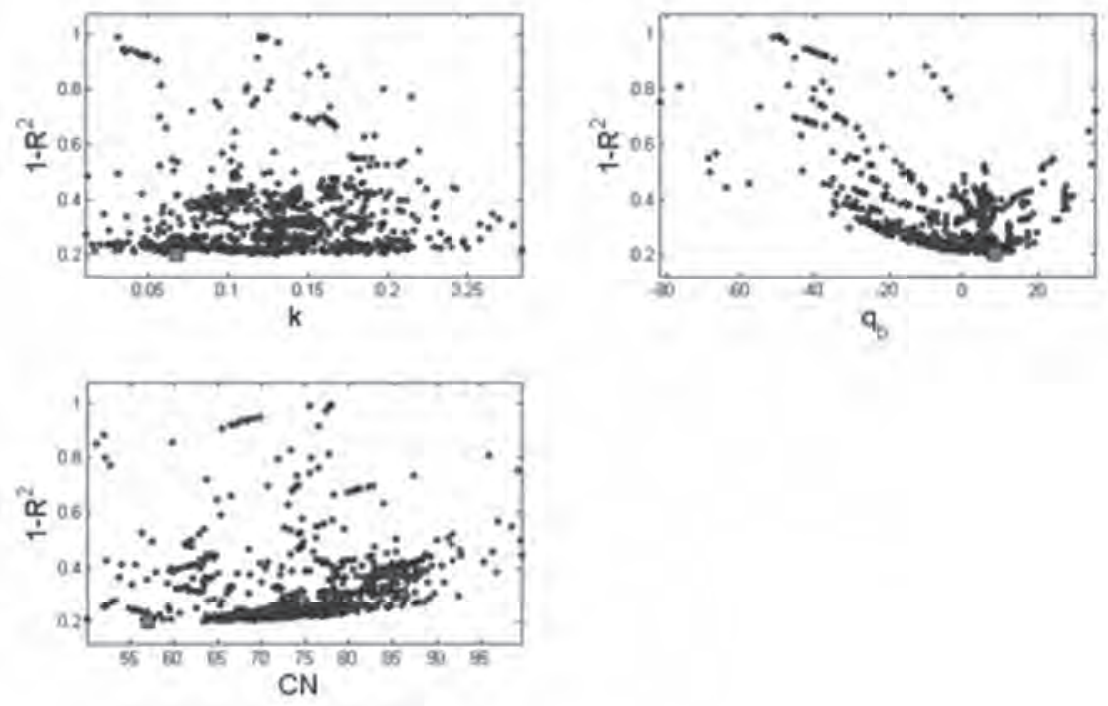

Figura 8. Diagrama de dispersión de los tres (3) parámetros del modelo del número de curva con su correspondiente medida de verosimilitud.

Fuente: Los autores

Por el comportamiento del diagrama de dispersión del parámetro del número de curva (CN), se ilustra una dinámica compleja durante su estimación con el algoritmo de calibración, pues se observa un espectro de valores agrupados dentro de un mismo margen de verosimilitud (por ejemplo: verosimilitud semejante para el rango definido entre $\mathrm{CN}=65$ y $\mathrm{CN}=80$ ). El mejor ajuste del procedimiento de calibración da como resultado un valor del CN igual a 57 que físicamente podría llegar a ser poco representativo de las condiciones predominantes del tipo y uso del suelo que se relaciona en la región de estudio.

\subsection{INTERACCIÓN DE LOS PARÁMETROS CN Y $\mathrm{Q}_{\mathrm{b}}$}

A partir de los resultados obtenidos con el procedimiento de calibración y del análisis de los parámetros, se puede evidenciar una relación biunívoca entre los parámetros $C N$ y $\mathrm{Q}_{\mathrm{b}^{\prime}}$ lo cual origina una expectativa conceptual muy importante. En la Figura 9, se puede apreciar una agrupación de los parámetros $\mathrm{CN}$ y $\mathrm{O}_{b}$ sobre una escala logarítmica. Por supuesto, para esta representación fue necesario desarrollar una simple transformación a los datos para poder representar los efectos de recarga hacia la fuente de flujo de agua subterránea. 


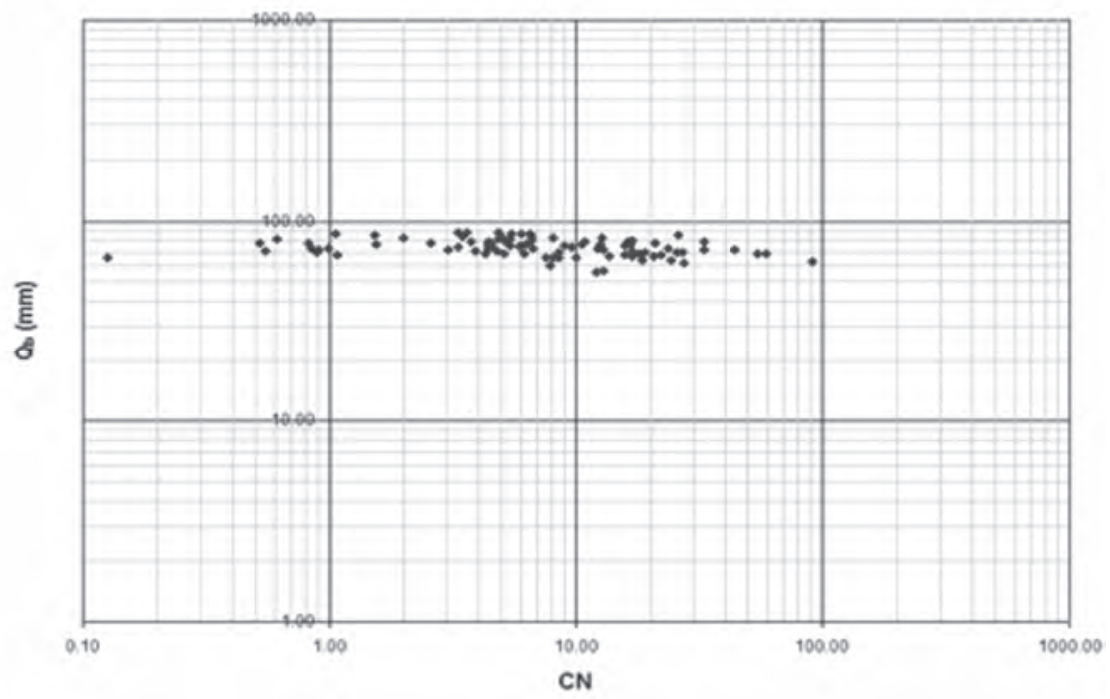

Figura 9. Relación entre los valores de los parámetros $\mathrm{CN}$ y $\mathrm{O}_{\mathrm{b}}$.

Fuente: Los autores

La Figura 9 ilustra un alineamiento moderadamente definido (con baja pendiente), entre los parámetros del modelo, lo cual redunda en el hecho de que podría existir una relación física entre las condiciones de tipo, uso y cobertura de suelo, con las condiciones físicas que determinan la dinámica del flujo de agua subterránea. Mediante un procedimiento de ajuste por mínimos cuadrados, se podría establecer una ecuación preliminar que represente la relación entre los parámetros para las condiciones del caso de estudio, tal como se presenta en la ecuación (7).

$$
\begin{array}{r}
Q_{b}=b \cdot C N^{m}-d \\
Q_{b}=2.5 \cdot C N^{0.6}-106.7
\end{array}
$$

Si se desconocen las fuentes de error del protocolo de modelación aquí indicado o bien, si se lograse extender este proyecto hasta la validación de la ecuación (7) para diferentes sistemas de cuencas hidrográficas; su aplicación traería grandes beneficios a los consultores y facilitaría la implementación de un modelo lluvia - escorrentía en sectores con limitada información. Sin embargo, también se puede apreciar que el modelo parsimonioso (de tres parámetros), propuesto inicialmente, tendría tres parámetros más que entrarían al procedimiento de calibración y podrían amplificar los niveles de incertidumbre del estado actual del modelo.

\subsection{INCERTIDUMBRE GENERAL DEL MODELO}

El procedimiento de calibración automática empleado en este proyecto, permitió establecer los mejores grupos de parámetros para representar el comportamiento del modelo y así mismo, 
establecer las bandas de incertidumbre de las realizaciones que puedan desarrollarse con la aplicación del modelo del número de curva. En la Figura 10, se demarcan estas bandas que tienen un comportamiento muy intrincado debido a la estructura misma de los datos de entrada. No obstante, la sensibilidad de las bandas de verosimilitud son considerablemente amplias y por lo tanto, establecen un alto margen de incertidumbre en los resultados del modelo.

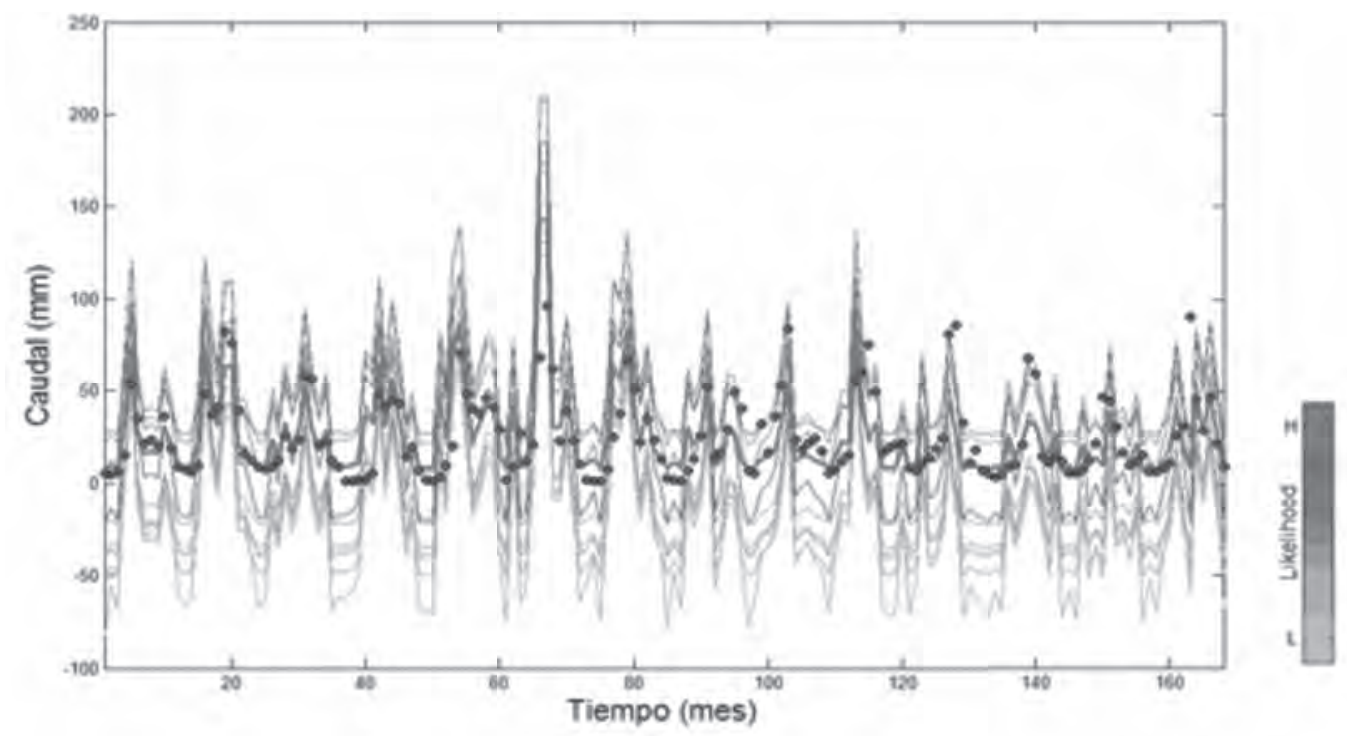

Figura 10. Demarcación de las bandas de confiabilidad del rendimiento general del modelo en función del grado de verosimilitud entre los valores observados (puntos sobre el gráfico), y los simulados.

Fuente: Los autores

\section{CONCLUSIONES}

Los fenómenos naturales son impredecibles y con su análisis se busca establecer valores que permitan la toma de decisiones para prevenir, controlar o mitigar los impactos que generen los valores extremos de tales eventos. En el caso de la modelación hidrológica, se plantean diferentes tipos de aproximaciones matemáticas que buscan representar mediante ecuaciones y el empleo de medios informáticos, el comportamiento de estos fenómenos para extrapolar sus efectos en el entorno natural o sobre una determinada comunidad.

En este contexto, para este proyecto se seleccionó una de las metodologías que se utilizan para establecer una relación lluvia - caudal de uso frecuente en una escala de tiempo determinada, pero poco creíble en una escala de tiempo diferente, como es el caso de la aplicación de la Teoría del CN. En esta fase del proyecto, se logró identificar los grupos de parámetros más aceptables para la aplicación del modelo del número de curva con la metodología de calibración y análisis e incertidumbre GLUE. Sin embargo, los resultados establecen a priori un problema notable de identificación de los parámetros que podría ser entendido bajo la tesis de equifinalidad. 
El modelo implica parámetros físicos de difícil cuantificación cuando se pretende interpretarlos en un entorno espacial, si se tiene en cuenta que la interpolación espacial no es precisa, y los resultados esperados posiblemente no sean los más satisfactorios. El empleo del Arc-Gis, por ejemplo, permite rasterizar una variable, 30 metros en este caso, con la facilidad de interpretar para cada celda, el valor de la precipitación, el tipo de suelo y por ende, su capacidad de infiltración, la cobertura vegetal y su potencial de retención, para estimar con ellos, la posible escorrentía directa esperada. Todo lo anterior, con base en la interpolación de los valores de las variables consideradas, por lo tanto, la precisión de los resultados soló será aproximada.

Aun cuando se han encontrado algunas dificultades en la estructura formal del modelo del número de curva, se puede aproximar una relación biunívoca entre los parámetros $C N$ y $\mathrm{Q}_{b^{\prime}}$, posibilitando una descripción más sencilla de los procesos de flujo de agua subterránea mediante la descripción física de las condiciones de uso y cobertura del suelo del sistema de cuenca que se modela.

Las escalas de tiempo en la evaluación de variables hidrológicas dependen en buena medida, del objetivo para el cual se desarrolla el análisis y la consistencia de la información de referencia. El flujo del agua en un medio poroso, es muy lento en comparación con la velocidad de desplazamiento de los flujos superficiales. Por lo tanto, cabría esperar que los aportes de aguas subterráneas a ese flujo superficial, sean moderadamente constantes en un determinado intervalo de tiempo; además, se debe considerar la localización de las zonas de recarga, pues es frecuente encontrar que el nivel freático en una zona determinada, se encuentre más profundo que el lecho del río, motivo por el cual también se debe considerar el aporte de la zonas de páramo y la influencia de los vertimientos de la comunidad vecina al río.

Este trabajo fue sólo una aproximación parcial al análisis de una metodología empleada para estimar el comportamiento de la relación lluvia - caudal en una escala de tiempo poco utilizada.

\section{AGRADECIMIENTOS}

Al Instituto Colombiano de Hidrología, Meteorología y Estudios Ambientales (IDEAM), y a la Corporación Autónoma Regional de Cundinamarca (CAR), por la información suministrada sobre la cuenca alta del río Bogotá, y que permitió el desarrollo de este proyecto.

\section{REFERENCIAS BIBLIOGRÁFICAS}

[1] Corredor J., (2005). Reflexiones sobre grandes crecientes en Colombia (inédito). Universidad Militar Nueva Granada, Bogotá. 
[2] Udall S.L.U., y Dominy F.E.U., (1979). Diseño de presas pequeñas. Méjico: Ediltorial Continental, México, pp. 459-460.

[3] Camacho L., (2006). Calibración y comparación de modelos de transporte de solutos en ríos de montaña. XXII Congreso Latinoamericano de Hidráulica, Guayana, Venezuela.

[4] Céspedes D, y Camacho L., (2002). Calibración, aplicación y comparación de modelos de flujo no permanente en la cuenca media del río Bogotá. (Tesis de grado), Facultad de Ingeniería, Universidad de lo Andes, Bogotá.

[5] Corredor J., (2006). El residuo líquido de las curtiembres. Estudio de Caso: Cuenca Alta del río Bogotá. En: Ciencia e Ingeniería Neogranadina, Vol. 16 (2), pp. 14-28.

[6] Universidad de Pamplona, (2010). Actualización de la oferta hídrica superficial para las cuancas hasta de quinto orden para la jurisdicción de la CAR. Corporación Autonóma Regional de Cundinamarca (CAR), Bogotá.

[7] Mockus V., (1978). Hydrology National Engineering. Natural Resources Conservation Service (USDA), Washington, $762 \mathrm{p}$.

[8] Mockus V., (1978). Hydrology National Engineering. Natural Resources Conservation Service (USDA), Washington, $762 \mathrm{p}$.

[9] Paz-Pellat F., (2009). Mitos y falacias del método hidrológico del Número de Curva del SCS/ NRCS. En: Agrociencia, Vol. 43 (5), pp. 521-528.

[10] López Cárdenas de Llano F.E., (1998). Restauración Hidrológico Forestal de Cuencas y Control de Erosión. Ministerio de Medio Ambiente, Tragsa, Tragsatec, Mundi Prensa, Madrid, pp. 112-115.

[1 1] Chow V., Maidment D., y Mays L., (1994). Hidrología Aplicada, McGraw-Hill. Bogotá.

[12] Wagener T., Lees M., and Wheater H., (2001). Montecarlo analysis toolbox user manual, London: Civil and Environmental Engineering Department, Imperial College of Science Technology and Medicine. 45 p.

[13] Wagener T., Freer J.E., Beven K.J., Gupta H.V., y Bardossy A., (2006). Toward and uncertainty framework for predictions in ungauged basins: The uncertainty working group, de Prediction in ungauged basin: promises and progress, Wallingford, Oxfordshire, Vol. 303, pp. 454-461.

[14] Beven K.J., (2005). A manifesto for the equifinality thesis. En: Journal of Hydrology, Vol. XX, pp. 1-19. 
[15] Beven K.J., (2001). Rainfall-runoff modeling: The primer West Sussex John \& Wiley, London, $359 \mathrm{p}$.

[16] Beven K.J., (2002). Towards a coherent philosophy for modelling the environment. En: Proceeding of Royal Society, London, Vol. 458, pp. 1-20.

[17] Willmott C., (1981). On the validation models. En: Journal of physical geography , Vol. 2, pp. 184-194.

[18] Beven K., (2009). Environmental modeling: an uncertain future. Ed. Routledge, Abingdon, $344 \mathrm{p}$.

[19] Camacho L., y Díaz-Granados M., (2003). Metodología para la obtención de un modelo predictivo de transporte de solutos y de calidad de agua en río - Caso río Bogotá, de la Hidroinformática en la Gestión Integrada de los Recursos Hídricos. Cartagena.

[20] Céspedes D., y Camacho L., (2004). Metodología de calibración de modelos hidrológicos e hidráulicos de flujo no permanente - Aplicación cuenca media del río Bogotá. XXI Congreso Latinoamericano de Hidráulica. San Pablo, Brasil.

[21] Aronica G., Candela A., Viola F., y Cannarozzo M., (2006). Influence of rating curve uncertainty on daily rainfall-runoff model prediction, Prediction in ungauged basin: promises and progress, Wallingford, Oxfordshire. Vol. 303, pp. 116-123.

[22] Mo X., Liu S., Lin X., Sun X., y Zhu Z., (2006). Multi-objetive conditioning of SVAT model for heat and $\mathrm{CO} 2$ fluxes predition, Prediction in ungauged basin: promises and progress, Wallingford, Oxforshire, Vol. 303, pp. 164-176.

[23] Romanowics R., Beven K.J., y Young P., (2006). Uncertainty propagation in a sequential model for flood forecasting, Prediction in ungauged basin: promises and progress. Wallingford, Oxfordshire, Vol. 303, pp. 177-184.

[24] Mujumdar P., Ghosh S., y Raje D., (2009). Hydrometeorological prediction from GCM simulations: downsacling techniques and uncertainty modelling, New approaches to hydrological prediction in data-sparse regions, IAHS. Vol. 333, pp. 165-175.

[25] Singh V., (1995). Computer models of watershed hydrology. Water Resources Publications, Colorado, $1130 \mathrm{p}$.

[26] Wagener T., Camacho L., y Wheater H.S., (2002). Dynamic identifiability analysis of the transient storage model for solute transport in rivers. En: Journal of Hydroinformatics, Vol. 04 (3), pp. 199-211. 
